\title{
Heavy-fermion and spin-liquid behavior in a Kondo lattice with magnetic frustration
}

\author{
S. Burdin ${ }^{1,2}$, D. R. Grempel ${ }^{3}$ and A. Georges ${ }^{4}$ \\ ${ }^{1}$ Département de Recherche Fondamentale sur la Matière Condensée, \\ SPSMS, CEA-Grenoble, 38054 Grenoble Cedex 9, France. \\ 2 Institut Laue-Langevin, B.P. 156, 38042 Grenoble Cedex 9, France. \\ 3 CEA-SACLAY, SPCSI, 91191 Gif-sur-Yvette Cedex, France. \\ ${ }^{4}$ CNRS - Laboratoire de Physique Théorique, Ecole Normale Supérieure, 24 Rue Lhomond 75005 Paris, France.
}

(October 30, 2018)

\begin{abstract}
We study the competition between the Kondo effect and frustrating exchange interactions in a Kondo-lattice model within a large- $\mathcal{N}$ dynamical mean-field theory. We find a $T=0$ phase transition between a heavy Fermi-liquid and a spin-liquid for a critical value of the exchange $J_{c}=T_{K}^{0}$, the single-impurity Kondo temperature. Close to the critical point, the Fermi liquid coherence scale $T^{\star}$ is strongly reduced and the effective mass strongly enhanced. The regime $T>T^{\star}$ is characterized by spin-liquid magnetic correlations and non-Fermi-liquid properties. It is suggested that magnetic frustration is a general mechanism which is essential to explain the large effective mass of some metallic compounds such as $\mathrm{LiV}_{2} \mathrm{O}_{4}$.
\end{abstract}

\section{INTRODUCTION}

The interplay between the Kondo effect and RKKY interactions is an essential feature of heavy-fermion systems. In dilute Kondo systems local magnetic moments are screened below the single-site Kondo temprrature $T_{K}^{0}$ and a local Fermi-liquid (FL) picture appliest. In dense systems, intersite magnetic interactions compete with the Kondo effect, leading to a quantum phase transition at which the metallic paramagnetic ground state becomes unstable when their strength attains a critical value $J_{c}$. In the vicinity of the quantum critical point (QCP) the physical properties of a large class of strongly correlated metals differ strikingly from those of normal Fermi liquids 3 .

The origin of non-Fermi liquid (NFL) behavior in the quantum critical region of heavy ferpion systems is an issue of current theoretical interest 1 . Two scenarios have been proposed for the case of the antiferromagnetic QCP5. In the first one, Kondo screening of the local moments takes place below a temperature $T_{K}$ that stays finite throughout the paramagnetic phase (including the QCP). At a lower coherence temperature $T^{\star}$, a heavy Fermi liquid of composite quasi-particles forms and the magnetic phase transition at $J_{c}$ is driven by a spin-density-wave (SDW) instability of the Fermi surface. NFL behavior around the QCP results from the coupling of the heavy electrons to critical long-wavelength SDW fluctuations 6 .

In the second scenario, intersite interactions are strong enough to prevent Kondo screening from occurring at the critical coupling. Both $T_{K}$ and $T^{\star}$ are expected to vanish at $J_{c}$, leading to the dissociation of the composite heavy quasi-particles into decoupled local moments and conduction electrons. In this case, NFL behavior is a consequence of the critical properties of the local spin fluctuations that are associated to the process of Kondo screening.
In systems with magnetic frustration, due either to the geometry of the lattice or to disorder, conventional magnetic ordering may give way to spin-glass (SG) freezing, or be suppressed altogether, leaving a correlated paramagnet or "spin-liquid" (SL) state.

In this paper we consider a Kondo-lattice model with frustrated magnetic interactions between the localized spins. We solve this model using a combination of dynamical mean-field theory and large- $\mathrm{N}$ techniques. We find a quantum critical point (QCP) between a Fermi liquid (FL) and a SL phase at a critical coupling $J_{c}=T_{K}^{0}$, the single-impurity Kondo temperature. We show that near the QCP the coupling of conduction electrons to local critical spin-fluctuations leads to the suppression of both the Kondo scale $T_{K}$ and of the FL coherence scale $T^{\star}$. In addition, $T_{K} / T^{\star} \gg 1$ for $J \sim J_{c}$, and the effective mass is drastically enhanced by the combined effect of the frustration and the Kondo effect near the QCP. This is reminiscent of the second scenario described above. However, it should be emphasized that the strong quantum fluctuations associated with the (fermionic) large-N limit considered here prevent any type of magnetic ordering to take place. As argued in the conclusion of the paper, corections beyond that limit will reintroduce (spin-glass) long-range order. At least in high enough dimensions, this will usually happen at a smaller value of the magnetic coupling $J$ than the one corresponding to the vanishing of the coherence scale. Nevertheless, the existence of a very small coherence scale and large effctive mass near the QCP, as well as that of an intermediate non-FL crossover regime between for $T^{*}<T<T_{K}$ are robust features. 


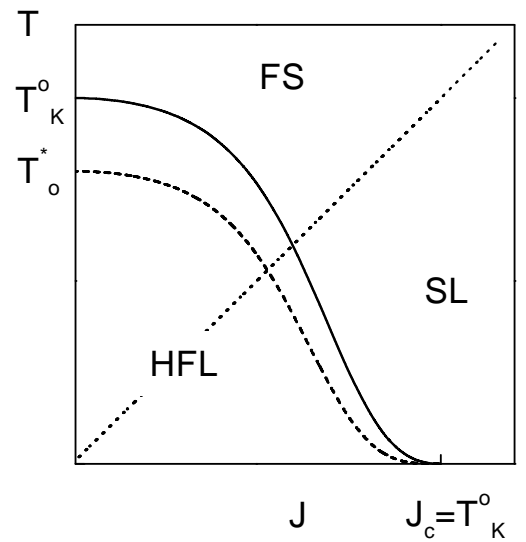

FIG. 1. Schematic phase diagram of the model in the $J-T$-plane. Kondo temperature (solid line) and coherence temperature (dashed line) as functions of $J$ for fixed values of $J_{K}$ and $n_{c}$. The system is a heavy Fermi liquid below $T^{\star}(J)$. Above the line $T_{K}(J)$, the localized spins are essentially free (FS) for $J<T$, while they form a highly correlated spin liquid for $J>T$. Near $J_{c}$ the spin liquid correlations start building up just above $T^{\star}$. All the lines represent crossovers.

Below $T^{\star}$ the low temperature properties of the system may be described in terms of heavy quasi-particles whose mass $m^{\star}$ diverges at the QCP. In the regime $T^{\star}<T<T_{K}$, the electrons form an incoherent "bad" metal. They gradually decouple from the localized spins for $T>T_{K}$, while the local spin dynamics remains SLlike for $T<J$. In the whole range $T^{\star}<T<J$, the spin and transport properties are markedly different from those of a FL. This type of non-Fermi liquid regime has been previously studied in Ref. 19 in the different context of a doped Mott insulator. As we shall see below, the physical properties of our model share common features with the experimentally observed behavior of $\mathrm{LiV}_{2} \mathrm{O}_{4}$, the first compound which displays heavy-fermion behavior even though no f-electrons are involved 10 .

\section{MODEL}

We study the Kondo-lattice model defined by the Hamiltonian :

$$
H=-\sum_{i, j, s} t_{i j} c_{i s}^{\dagger} c_{j s}+J_{K} \sum_{i} \vec{S}_{i} \cdot \vec{s}_{i}+\sum_{<i, j>} J_{i j} \vec{S}_{i} \cdot \vec{S}_{j}
$$

where $\vec{S}_{i}$ and $c_{i s}^{\dagger}$ represent respectively a localized spin and a conduction-electron creation operator at the $i$-th site of the lattice. The localized spins interact with the conduction-electron spin density $\vec{s}_{i}=$ $1 / 2 \sum_{s, s^{\prime}} c_{i s}^{\dagger} \vec{\sigma}_{s s^{\prime}} c_{i s^{\prime}}$ via a local Kondo coupling $J_{K}$ and $J_{i j}$ is the magnetic exchange coupling between nearestneighbor pairs of localized spins. The effects of frustration are introduced in the model by an appropriate choice of the couplings $J_{i j}$. In a model for geometrically frustrated materials these must be taken anti-ferromagnetic on lattices of the Kagome or pyrochlore typed. In the case of a metallic SG, the couplings can be taken as a set of random variables with zero mean and variance $J / \sqrt{z}$ ( $\mathrm{z}$ is the coordination of the lattice). In the following, we focus on the case of random $J_{i j}$. This should be viewed simply as a way to generate a SL regime with non-trivial spin dynamics, and we expect our conclusions to be similar in the case of geometric frustration.

The problem defined by Eq. (1) is tractable in the limit of large coordination $z \rightarrow \infty$ where dynamical mean-field theory (DMFT) is applicable11 and the model can be reduced to an effective single-site theory. Using standard methods to perform the average over the disorder 12 the DMFT action associated to Eq. (1) may be written in the form 13 :

$$
\begin{aligned}
\mathcal{A}= & \sum_{s} \int_{0}^{\beta} d \tau \int_{0}^{\beta} d \tau^{\prime} c_{s}^{\dagger}(\tau)\left[\left(\partial_{\tau}-\mu\right) \delta\left(\tau-\tau^{\prime}\right)\right. \\
& \left.-t^{2} G_{c}\left(\tau-\tau^{\prime}\right)\right] c_{s}\left(\tau^{\prime}\right)+J_{K} \int_{0}^{\beta} d \tau \vec{S}(\tau) \cdot \vec{\sigma}_{c}(\tau) \\
& -\frac{J^{2}}{2} \int_{0}^{\beta} d \tau \int_{0}^{\beta} d \tau^{\prime} \chi\left(\tau-\tau^{\prime}\right) \vec{S}(\tau) \cdot \vec{S}\left(\tau^{\prime}\right)
\end{aligned}
$$

where $\vec{\sigma}_{c}=\sum_{s, s^{\prime}} \vec{\sigma}_{s s^{\prime}} c_{s}^{\dagger}(\tau) c_{s^{\prime}}(\tau)$ is the conduction electron spin density, $\mu$ is the chemical potential and the rescaling $t \rightarrow t / \sqrt{z}$ was performed in order to obtain finite expressions in the limit $z \rightarrow \infty . G_{c}(\tau)$ and $\chi(\tau)$ are the conduction-electron Green function and the localized spin autocorrelation function, respectively. These are determined by the self-consistency condition 11,12 $G_{c}(\tau)=-\left\langle c_{s}(\tau) c_{s}^{\dagger}(0)\right\rangle_{\mathcal{A}}$ and $\chi(\tau)=\langle\vec{S}(\tau) \cdot \vec{S}(0)\rangle_{\mathcal{A}}$. The first two terms in Eq. (2) represent the action of the Kondo-lattice hamiltonian. The last term is the action of the quantum SG model recently studied by several authors 14 16. It was shown that for $S=1 / 2$ the ground state has SG order 15 . However, SL solutions appear above the SG transition temperatyre, specially in the formal limit of small values of $S 44$. 3 . Furthermore, it has been recently suggested 17 that the effective action describing the spin dynamics in this regime is also relevant for geometrically frustrated antiferromagnets.

The SL solutions are well described in the large- $\mathcal{N}$ approach that we discuss next.

\section{LARGE- $\mathcal{N}$ SOLUTION}

The model defined in Eq. (2) cannot be solved analytically as it stands, but much progress can be made by solving it in the large- $\mathcal{N}$ approach which has been extensively used in the study of the Kondo latticets and also allows to deal with the magnetic exchange term in Eq. (2) $14,16.19$. In this approach the spin symmetry is extended to $\mathrm{SU}(\mathcal{N})$ and the coupling constants are rescaled as $J_{K} \rightarrow J_{K} / \mathcal{N}$, $J \rightarrow J / \sqrt{\mathcal{N}}$. The localized spin is represented in terms of fermion operators, $S^{\sigma \sigma^{\prime}}=f_{\sigma}^{\dagger} f_{\sigma^{\prime}}-\delta_{\sigma \sigma^{\prime}} / 2$, subject to the 
local constraint $\sum_{\sigma} f_{\sigma}^{\dagger} f_{\sigma}=\mathcal{N} / 2$. The interaction terms in Eq. (2) become now quartic. These are decoupled introducing Hubbard-Stratonovich fields $B(\tau)$ (conjugate to $\sum_{\sigma} f_{\sigma}^{\dagger} c_{\sigma}$ ) and $P\left(\tau, \tau^{\prime}\right)$ (conjugate to $\sum_{\sigma} f_{\sigma}^{\dagger} f_{\sigma}$ ) and the constraint is enforced through the introduction of a Lagrange multiplier $i \lambda(\tau)$. In the $\mathcal{N} \rightarrow \infty$ limit the physics is controlled by a saddle point at which the Bose field condenses $\langle B(\tau)\rangle_{\mathcal{A}}=r$, and the Lagrange multiplier takes a static value $i \lambda(\tau)=\lambda$, while $P\left(\tau, \tau^{\prime}\right)=P\left(\tau-\tau^{\prime}\right)$ generates a frequency dependent local self-energy 14.6 . The saddle point equations can be written in the following compact form:

$$
\left\{\begin{array}{c}
-r / J_{K} \\
1 / 2 \\
n_{c} / 2
\end{array}\right\}=-\frac{1}{\pi} \int_{-\infty}^{\infty} d \omega n_{F}(\omega) \operatorname{Im}\left\{\begin{array}{c}
G_{f c}(\omega) \\
G_{f}(\omega) \\
G_{c}(\omega)
\end{array}\right\},
$$

where $n_{F}$ is the Fermi function and $G_{c}, G_{f}$ and $G_{f c}$ are the full conduction-electron, f-electron and mixed Green functions, respectively given by :

$$
\begin{aligned}
G_{c}(\omega) & =G_{c}^{0}\left(\omega+\mu-r^{2} \mathcal{G}_{f}(\omega)\right) \\
G_{f}(\omega) & =\mathcal{G}_{f}(\omega)\left[1+r^{2} \mathcal{G}_{f}(\omega) G_{c}(\omega)\right] \\
G_{f c}(\omega) & =r \mathcal{G}_{f}(\omega) G_{c}(\omega) .
\end{aligned}
$$

Here, $G_{c}^{0}(\omega)=\sum_{k} 1 /\left(\omega-\epsilon_{k}\right)$ is the non-interacting electronic local Green function, and we introduced the "bare" f-electron Green function

$$
\mathcal{G}_{f}(\omega)=\frac{1}{\omega+\lambda-\Sigma_{l o c}(\omega)},
$$

where the local self-energy is

$$
\Sigma_{l o c}(\tau)=-J^{2} G_{f}^{2}(\tau) G_{f}(-\tau) .
$$

\section{A. The Kondo temperature}

At high temperature or for large values of $J$ the only solution of Eqs. (3)-(6) has $r=\lambda=0$. This represents a regime in which the localized spins and the conduction electrons are decoupled. In this regime $\mathcal{G}_{f}(\omega)=G_{S}(\omega)$, the solution of a non-linear integral equation first investigated by Sachdev and Ye 14 that reads:

$$
\begin{aligned}
G_{S}\left(i \omega_{n}\right) & =\left[i \omega_{n}-\Sigma_{S}\left(i \omega_{n}\right)\right]^{-1} \\
\Sigma_{S}(\tau) & =-J^{2}\left[G_{S}(\tau)\right]^{2} G_{S}(-\tau) .
\end{aligned}
$$

In the region $\max (T, \omega)<J$ the solution of this solution describes a SL with a non-trivial local spin dynamics characterized by a slow decay of the local spin autocorrelation function $14,\langle\vec{S}(0) \cdot \vec{S}(t)\rangle \sim 1 / t$.

In the large- $\mathcal{N}$ theory, the onset of Kondo screening is signaled by a phase transition at a critical temperature $T_{K}$ at which a second solution with $r \neq 0$ appears. The equation for $T_{K}$ is thus

$$
\frac{1}{J_{K}}=\int_{-\infty}^{\infty} \frac{d \omega}{\pi} n_{F}(\omega) \operatorname{Im}\left[G_{c}^{0}(\mu+\omega) G_{S}(\omega)\right],
$$

where $\mu$ is the chemical potential for free conduction electrons with density $n_{c}$ at $T=T_{K}$. In the limit $J \rho_{0}(\mu) \ll 1$ this equation can be cast in the form

$$
\begin{aligned}
\frac{1}{J_{\mathrm{K}}} & =-\frac{\rho_{0}(\mu)}{2} \int_{-\infty}^{\infty} d \epsilon\left(G^{\prime}{ }_{S}(\epsilon)-\frac{1}{\epsilon}\right) \tanh \left(\frac{\epsilon}{2 T_{K}}\right) \\
& +\frac{1}{2} \int_{-\infty}^{\infty} \frac{d \epsilon}{\epsilon} \rho_{0}(\mu+\epsilon) \tanh \left(\frac{\epsilon}{2 T_{K}}\right)
\end{aligned}
$$

where $G^{\prime}{ }_{S}$ denotes the real part of the f-electron Green function and $\rho_{0}$ is the conduction electron density of states (d.o.s).

For $J \rightarrow 0, G_{S}(\omega)=\omega^{-1}$ and only the second integral on the right-hand side of Eq. (10) survives. Then we find that $T_{K}=T_{K}^{0}$, the single-impurity Kondoscale, given in the weak-coupling limit $J_{K} \rho_{0}\left(\epsilon_{F}\right) \ll 1$ by 20 :

$$
T_{K}^{0}=D e^{-1 /\left(J_{K} \rho_{0}\left(\epsilon_{F}\right)\right)} \sqrt{1-\left(\epsilon_{F} / D\right)^{2}} F_{K}\left(n_{c}\right)
$$

where $\epsilon_{F}$ is the non-interacting Fermi level and

$$
\ln F_{K}\left(n_{c}\right)=\int_{-\left(D+\epsilon_{F}\right)}^{D-\epsilon_{F}} \frac{d \epsilon}{|\epsilon|} \frac{\rho_{0}\left(\epsilon_{F}+\epsilon\right)-\rho_{0}\left(\epsilon_{F}\right)}{2 \rho_{0}\left(\epsilon_{F}\right)},
$$

depends on the details of the band structure and the filling of the conduction band but not on the Kondo couplingen.

For $J \ll T_{K}^{0}$ the intersite coupling is a small perturbation and it may be shown that $T_{K}=$ $T_{K}^{0}\left[1-\mathcal{O}\left(\left(J / T_{K}^{0}\right)^{2}\right)\right]$. In the opposite limit, $J \gg T_{K}^{0}$, the magnetic exchange dominates and it can be shown that the form of the decay of the spin autocorrelation function in the SL phase implies that the Kondo effect cannot take place. As a result, for $J$ above a critical value $J_{c}=T_{K}^{0}$, the conduction electrons remain decoupled from the localized spins down to zero temperature (Fig. 1). This is expected from a comparison of the binding energy of two localized spins in the SL regime $(\sim J)$ and the energy gained by forming singlets between the spins and the conduction electrons which is at most $\mathcal{O}\left(T_{K}^{0}\right)$.

In order to find the behavior of $T_{K}$ close to the critical point, we evaluate the first integral on he right-hand side of Eq. (10) using the asymptotic form 19 of $G_{S}(\omega)$,

$$
G_{S}(\omega) \sim\left\{\begin{array}{cl}
\frac{\Gamma[1 / 4]^{2}}{2 \pi^{5 / 4}} \frac{\omega /(2 T)-i}{\sqrt{J T}}, & |\omega|<T \ll J, \\
\left(\frac{\pi}{4}\right)^{1 / 4} \frac{\operatorname{sign}(\omega)-i}{\sqrt{J|\omega|}}, & T<|\omega| \ll J .
\end{array}\right.
$$

To leading order in $T_{K} / J$ we find:

$$
I_{1} \sim \rho_{0}(\mu)\left[\ln \left(T_{K} / J\right)-a \sqrt{T_{K} / J}\right],
$$

where $a$ is a numerical constant of $\mathcal{O}(1)$. Combining Eqs. (10), (11) and (14) we find that, for $J \sim J_{c}=T_{K}^{0}$, 


$$
T_{K} \approx J \ln ^{2}\left(\frac{T_{K}^{0}}{J}\right) \sim T_{K}^{0} \delta^{2}
$$

where $\delta=\left(J_{c}-J\right) / J_{c}$ measures the distance to the QCP where the Kondo temperature vanishes quadratically.

\section{B. The heavy Fermi-liquid regime}

When $r$ is finite, Eqs. (3)-(6) admit FL solutions at low enough temperatures. We discuss first the case $T=0$. A calculation analogous to that performed in Ref. 19 yields the value of the self-energy at zero-frequency :

$$
r^{2} /\left(\Sigma_{l o c}(0)-\lambda\right)=\epsilon_{F}^{>}-\mu,
$$

In this expression, $\epsilon_{F}^{>}$is the non-interacting Fermi level corresponding to an electron density $\left(n_{c}+1\right) / 2$ per spin component. Eq. (16) implies that Luttinger's theorem is satisfied, with a "large" Fermi surface containing both conduction electrons and localized spins. In the weakcoupling limit, $\mu \approx \epsilon_{F}$, the non-interacting Fermi level corresponding to a density $n_{c}$. It can be shown from Eqs. (4), (6) and (16) that the f-electron d.o.s at the Fermi level $\rho_{f}(0)=\mathcal{O}\left(D / r^{2}\right)$ is finite for $r \neq 0$. Since in the SL phase $\rho_{f}(\omega) \propto 1 / \sqrt{J|\omega|}$, a crossover between the FL and SL regimes is expected at a scale $T^{\star} \sim\left(r^{2} / D\right)^{2} / J$. We note that, for $J=0$, the coherence scale that contrels all physical quantities at low temperature is $r^{2} / D 20$. For $J \neq 0$, this role is played by the much smaller scale $T^{\star}$, as detailed below.

We found that, at $T=0$, in the weak-coupling limit, the full set of Eqs. (3)-(6) can be solved analytically in the vicinity of the QCP by using the following Ansatz for the f-electron d.o.s :

$$
\rho_{f}(\omega)=\left\{\begin{array}{ll}
\left(\epsilon_{F}^{>}-\epsilon_{F}\right)^{2} \rho_{0}\left(\epsilon_{F}^{>}\right) / r^{2} & \text { for } \omega \leq T^{\star} \\
\left(4 \pi^{3}\right)^{-1 / 4}(J|\omega|)^{-1 / 2} & \text { for } T^{\star}<\omega<J
\end{array} .\right.
$$

The first line in the above equation is $\rho_{f}(0)$ as determined from Eqs,(4) and (16); the second line is the SL density of states 44 and $T^{\star}$ is defined as the energy at which the two expressions match, i.e.:

$$
T^{\star} \propto \frac{1}{J}\left(\frac{r^{2}}{\left(\epsilon_{F}^{>}-\epsilon_{F}\right)^{2} \rho_{0}\left(\epsilon_{F}^{>}\right)}\right)^{2} .
$$

This establishes a relationship between $T^{\star}$ and $r$ that is determined from the equation

$$
\frac{1}{J_{K}}=\int_{-\infty}^{0} \frac{d \omega}{\pi} \operatorname{Im}\left[\mathcal{G}_{f}(\omega) G_{c}^{0}\left(\omega-r^{2} \mathcal{G}_{f}(\omega)\right)\right] .
$$

The bare f-Green function $\mathcal{G}_{f}(\omega)$ may be computed using Eqs. (4), (6) and (17) and the integral in Eq. (19) may be evaluated in the limit $J \rightarrow J_{c}$. We find :

$$
T^{\star} \sim T_{K}^{0} \delta^{2} /(\ln \delta)^{2} .
$$

$T^{\star}$ and $T_{K}$ thus vanish simultaneously at the critical coupling, with $T_{K} / T^{\star} \sim \ln ^{2} \delta \gg 1$ as $\delta \rightarrow 0$.

The various physical regimes that follow from these considerations are depicted schematically in Fig. 1. Above the scale $T_{K}(J)$, the conduction electrons and the localized spins are decoupled. This is clearly an unrealistic feature of the large- $\mathcal{N}$-limit. For finite $\mathcal{N}$, the phase transition at $T_{K}$ will be replaced by a gradual decoupling of the electrons and the spins as the temperature is raised. For $T_{K}<T<J$, the localized spins remain strongly correlated in the SL state. For $T<T^{\star}$, a heavy Fermi liquid with a large Fermi surface is formed. The effective mass of quasiparticles (given by the inverse of the quasiparticle residue $Z$ ) is $m^{*} / m=1 / Z \sim D / T^{\star} \sim D / T_{K}^{0}(\ln \delta / \delta)^{2}$. This is one of the key results obtained in this paper: it demonstrates how the frustrating magnetic exchange leads to a dramatic enhancement of the effective mass with respect to the "bare" Kondo scale. The underlying mechanism is the large entropy of the SL state at low temperature. The intermediate range $T^{\star}<T<T_{K}$ corresponds to a crossover regime in which, as the temperature decreases, the electrons gradually couple to the localized spins, and the spin correlations change from SL-like at high temperature to FL-like at low temperature.

\section{PHYSICAL PROPERTIES}

The physical properties of the system can be computed in a standard way from the Green functions. We find that, for $T \ll T^{\star}$, the entropy is dominated by the quasiparticle contribution, $S \propto T \rho_{f}(0)\left(1-\partial \Sigma(\omega) /\left.\partial \omega\right|_{\omega=0}\right)$. Each of last the two factors gives a contribution proportional to $\left(J / T^{\star}\right)^{1 / 2}$. Thus, in the FL region $C \propto T / T^{\star}$. (17) In the SL regime for $T^{\star}<T \ll J$ we find $C \propto \sqrt{T / J}$. The specific heat thus has a peak at $T \sim T^{\star}$.

In the Fermi-liquid region we find the local spin susceptibility $\chi_{l o c}^{\prime \prime}(\omega) \propto \omega / T^{\star}$. At $T \sim T^{\star}$ there in crossover to the SL form $J \chi_{l o c}^{\prime \prime}(\omega) \propto \tanh \omega / 2 T 44.19$. Hence, the NMR spin-lattice relaxation rate $1 / T_{1} \propto$ $T \lim _{\omega \rightarrow 0} \quad \chi_{l o c}^{\prime \prime}(\omega) / \omega$ obeys Korringa's law $1 / T_{1} \sim$ $T /\left(J T^{\star}\right)$ below $T=T^{\star}$, but is $T$-independent above this temperature, $1 / T_{1} \sim 1 / J$. The static local susceptibility increases logarithmically with decreasing temperature for $T>T^{\star}, \chi_{l o c}(T) \propto J^{-1} \ln (J / T)$, saturating to a constant value $\propto J^{-1} \ln \left(J / T^{\star}\right)$ for $T<T^{\star}$. In analogy with the result found in the closely related case of the doped Mott insulator 19 we expect a finite uniform spin $\chi=\mathcal{O}\left(J^{-1}\right)$ at loy temperature .

Within DMFTL1, the dc resistivity is easily obtained from one-particle properties since vertex corrections are absent. In the Fermi-liquid regime $T<T^{\star}$, we find $\rho_{d c}(T) \propto\left(T / T^{\star}\right)^{2}$ : hence the Kadowaki-Woods relation is obeyed as in most heavy-fermion compounds. In the intermediate regime $T^{\star}<T<T_{K}$, the resistivity drops as 
$\rho_{d c}(T) \propto\left(T^{\star} / T\right)^{1 / 2}$. Although reminiscent of the maximum observed in usual heavy-fermion systems the physical origin of this feature in the present case is different. Here, the localized spins form tightly bound singlet pairs in the SL phase at $T^{\star} \ll T \ll J$. Hence, the correlations between the localized spins increase as $T$ increases from $T^{\star}$, which results in the decrease of the scattering cross section of conduction electrons. However, residual scattering of the conduction electrons on the local spin fluctuations of the SL is still expected to contribute to the resistivity for $T_{K}<T<J$. The discussion of this effect requires going beyond the $\mathcal{N} \rightarrow \infty$ limit (while we do not expect qualitative changes in the FL region from the inclusion of higher order corrections). There is in particular a second-order correction to the conduction electron self-energy that reads:

$$
\operatorname{Im} \Sigma_{c}(0) \propto\left(J_{K} / \mathcal{N}\right)^{2} \rho_{0}(0) \int_{0}^{\infty} \frac{d \omega}{\sinh (\beta \omega)} \chi_{l o c}^{\prime \prime}(\omega) .
$$

Since $\chi_{l o c}^{\prime \prime}(\omega) \propto \omega / T$ for $\omega<T$ and $T>T^{\star}$, this results in a contribution $\delta \rho \propto T$ to the resistivity. We thus expect to see in the physical case $\mathcal{N}=2$ a crossover from a quadratic to a linear $T$-dependence of the resistivity at $T \approx T^{\star}$.

\section{CONCLUSIONS}

In summary, we studied a model in which the competition between the Kondo effect and frustrating magnetic interactions leads to a QCP separating a heavy Fermiliquid phase from a spin-liquid phase. The coupling of the conduction electrons to critical local spin-fluctuations near the QCP results in a dramatic reduction of both the Kondo temperature and the Fermi-liquid coherence scale, and to a critically enhanced effective mass. For temperatures above the FL coherence scale but below the magnetic exchange, the transport and spin dynamics show striking deviations from those of a Fermi liquid.

To conclude this paper, we would like to make several remarks regarding the possible physical relevance of this model, and of our findings.

First, it is worth mentioning that some of the results derived here are independent of the specific form of the kernel $\chi(\tau)$ but results from the existence of an unstable fixed point that lies between the Kondo phase and the spin fluctuation dominated phase as pointed out in 21 . Some of our conclusions are therefore expected to be valid beyond our specific model, as long as the time-decay of the local spin correlations is sufficiently slow.

Next, we would like to comment on the manner in which long-range magnetic order (LRO) can possibly affect our results. In the solution presented in this paper, spin-glass ordering does not appear because it is suppressed by the strong quantum fluctuations associated with the fermionic representation of spins in the large- $\mathcal{N}$ limit16. Taking LRO into account in the present model thus requires either to consider bosonic representations 16 (which however makes the Kondo effect more difficult to treat technically) or to go beyond large- $\mathcal{N}$ in the fermionic case. It is clear that spin-glass ordering will show up at first order in the $1 / \mathcal{N}$ expansion, in the meanfield (infinite connectivity) model. One can quantify this by noting that the criterion for the spin-glass transition reads 16: $J \chi_{l o c}(T, J) \propto \sqrt{\mathcal{N}}$. In our solution, $\chi_{l o c}$ is proportional to $1 / T^{*}$, which is given by (20) near the QCP. Using this expression, we see that LRO should set in at a value of $J$ which is smaller than the critical value $J_{c}$ found above (at which the coherence scale vanishes): $J_{L R O}=J_{c}\left(1-c / \mathcal{N}^{1 / 4}\right)$. Hence, we expect that, in high enough dimensions (where mean-field theory applies), the vanishing of the Kondo scale will be preempted by magnetic LRO. Nevertheless, some of the qualitative features found above may still be important in practice when the coherence scale is small enough at the transition point. The situation in low dimensions, when spatial fluctuations are stronger, is quite open. Whether it is possible to reach the "coherence" transition before LRO sets in, as suggested in Ref. 5 is a fascinating question.

Finally, we would like to address the qualitative relevance of some of the findings of this paper for the physics of $\mathrm{LiV}_{2} \mathrm{O}_{4}$ and other compounds such as $\mathrm{YScMn}_{2}$ and $\beta$ Mn. $\mathrm{LiV}_{2} \mathrm{O}_{4}$ has a structure in which the $\mathrm{V}$ ions form a lattice of corner-sharing tetrahedra. This highly frustrated structure is kpown to lead to unconventipmal spinliquid ground-statest and recent NMR studie 22 indeed showed evidence of the presence of SL-like spin correlations in $\mathrm{LiV}_{2} \mathrm{O}_{4}$. This system is also close to a SG instability as it is known that small ampunts of $\mathrm{Zn}$ doping on the Li sites results in SG freezing 23 According to LDA $+\mathrm{U}$ band structure calculations 2425 one of the three $t_{2 g}$ $\mathrm{V}$ d-levels splits from the triplet in the crystal field of $\mathrm{LiV}_{2} \mathrm{O}_{4}$ and forms a highly correlated band. Due to the large Coulomb repulsion on the $\mathrm{V}$ site the corresponding Wannier states are singly-occupied thus playing a role similar to that of the f-orbitals in conventional rare-earth based heavy-fermion systems.

In Ref. 24, a Kondo lattice model was thus proposed to describe $\mathrm{LiV}_{2} \mathrm{O}_{4}$. This model in fact neglects the important ferromagnetic on-site Hund's coupling between the itinerant and localised orbital28. Let us however comment on the estimate of the resulting coherence temperature made in Ref. 24. The single-site Kondo temperature estimated there from the electronic structure calculations is $T_{K}^{0} \approx 550 \mathrm{~K} 24$. This is an order of magnitude larger than the measured Fermi liquid coherence temperature $T^{\star} \approx 25-40 \mathrm{~K}$. Nozière's "exhaustion" mechanism 26 was invoked to explain this huge reduction of $T^{\star}$ with respect to $T_{K}^{0}$. However, some doubts where recently casted.27 on the validity of Nozières' estimate $T^{\star} \propto\left(T_{\mathbb{A}^{*}}^{0}\right)^{2} / D(D$ is the bandwidth $)$ and it has been suggested 20 that $T^{\star} \propto T_{K}^{0}$ with a prefactor that is small only for very low values of the conduction-electron den- 
sity, a situation that is not realized in $\mathrm{LiV}_{2} \mathrm{O}_{4}$. Hence, an alternative mechanism is needed in order to explain the observed reduction of $T^{\star}$. As shown in this paper, magnetic frustration does provide such a mechanism in a Kondo lattice model.

However, as pointed out in Ref. 28, it is crucial to take into account the Hund's rule coupling in a realistic modelling of $\mathrm{LiV}_{2} \mathrm{O}_{4}$ and related compounds. Hence the results of the present paper cannot be straightforwardly applied to these compounds. However, we observe that the strong mass enhancment due to frustration has a simple qualitative origin: it is due to the storage of entropy at low-temperature associated with the spin-liquid state characteristic of frustrated systems. Hence, we expect that other models in which electrons are strongly coupled to local spins with SL dynamics will also lead to such a mass enhancment and suppression of the coherence scale. In $\mathrm{LiV}_{2} \mathrm{O}_{4}$, the $T$-independent spin relaxation rate for $T>T^{\star}$ as well as incoherent metallic transport $\rho \propto T$ in this regime is, in our opinion, strong experimental evidence that the local spin dynamics characteristic of a frustrated spin-liquid is playing a key role in this system.

\section{ACKNOWLEDGMENTS}

We thank C. Lacroix for useful comments and discussions and N. Buettgen for making Ref. 22 available to us prior to publication. A.G acknowledges early discussions with A. Sengupta and O. Parcollet on closely related subjects as well as useful discussions with H. Takagi, V. Anisimov and M. Katsnelson on $\mathrm{LiV}_{2} \mathrm{O}_{4}$.

${ }^{1}$ See, e.g.,A. C. Hewson, The Kondo problem to heavy fermions, Cambridge University Press, Cambridge, (1993).

2 S. Doniach, Physica B 91, 231 (1977).

${ }^{3}$ M. B. Maple et al., J. Low Temp. Phys. 95, 225 (1994); G. Steward, Rev. Mod. Phys., in press (2001).

${ }^{4}$ P. Coleman, C.Pepin, Q. Si and R. Ramazashvili, J. Phys. Cond. Mat 13, R723 (2001).

${ }^{5}$ Q. Si, S. Rabello, K. Ingersent and J. Lleweilun Smith, Nature 413, 804 (2001).

${ }^{6}$ J. A. Hertz, Phys. Rev. B 14, 1165 (1976); A. J. Millis, Phys. Rev. B 48, 7183 (1993).

${ }^{7}$ P. Schiffer and A. P. Ramirez, Comments Condens. Matter Phys. 18,21 (1996).

${ }^{8}$ S. Kondo et al., Phys. Rev. Lett. 78, 3729 (1997).

${ }^{9}$ H. Kusunose, S. Yotsuhashi and K. Miyake, Phys. Rev. B 62, 4403 (2000).

10 S.-H. Lee et al., cond-mat/0102414, (unpublished).

11 A. Georges et al. Rev. Mod. Phys. 68, 13 (1996).

12 A. J. Bray and M. A. Moore, J. Phys. C 13, L655 (1980).
13 The explicit form of the last term in the electronic part of the action in Eq. (2) corresponds to the choice of a semi-circular conduction-band density of states, $\rho_{0}(\epsilon)=$ $2 / \pi \sqrt{1-\epsilon^{2} / D^{2}}$. The results that follow are qualitatively independent of the detailed form of $\rho(\epsilon)$.

${ }^{14}$ S. Sachdev and J. Ye, Phys. Rev. Lett. 70,3339 (1993).

${ }^{15}$ D. R. Grempel and M. J. Rozenberg, Phys. Rev. Lett. 80 389, (1998).

16 A. Georges, S. Sachdev and O. Parcollet, Phys. Rev. Lett. 85, 840 (2000); Phys. Rev. B, 63134406 (2001).

17 A. Georges, R. Siddharthan and S. Florens , Phys. Rev. Lett. 87277203 (2001).

18 See e.g. for a review: D. M. Newns and N. Read Adv. Phys. 36, 799 (1987).

19 O. Parcollet and A. Georges, Phys. Rev. B 59,5341 (1999).

${ }^{20}$ S. Burdin, A. Georges and D. R. Grempel Phys. Rev. Lett. 85, 1048 (2000).

${ }^{21}$ A. M. Sengupta , Phys. Rev. B 61, 4041 (2000).

${ }^{22} \mathrm{~N}$. Buettgen et al., Proceedings of the HFM-Conference. June 2000, Waterloo, Ontario,Canada. To appear in Can. J. Phys., (2001).

${ }^{23}$ N. Fujiwara, H. Yasuoka and Y. Ueda, Phys. Rev. B

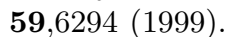

${ }^{24}$ V. I. Anisimov et al., Phys. Rev. Lett. 83, 364 (1999).

${ }^{25}$ V. Eyert et al., Europhys. Lett. 46, 762 (1999) .

${ }^{26}$ P. Nozières , Ann. Phys. Fr. 10,19 (1985); Eur. Phys. B, 6 (1998) 447.

27 Th. Pruschke et al., Phys. Rev. B 61, 12799 (2000).

${ }^{28}$ C. Lacroix, Proceedings of the HFM-Conference. June 2000, Waterloo, Ontario,Canada. preprint cond-mat 0107574, to appear in Can. J. Phys., (2001). 